\title{
A Robust Power Allocation Scheme in Ad-Hoc Cognitive Radio Networks
}

\author{
https://doi.org/10.3991/ijoe.v13i08.6906 \\ Hongzhi Wang, Meng Zhu, Mingyue Zhou( $\left.{ }^{\varpi}\right)$ \\ Changchun University of Technology, Changchun, China \\ zmyjlu@ccut.edu.cn
}

\begin{abstract}
We investigate the secondary users (SUs) power allocation in adhoc cognitive radio networks, considering an underlay paradigm where SUs can access the licensed spectrum owned by the primary users (PUs), if the total power to SUs and the interference power threshold from PUs and the signal-tointerference-noise-ratio (SINR) requirements of SUs are guaranteed. While prevalent works about distributed resources allocation algorithms are mainly focused on power allocation under the consideration of many practical limitations, which may not be the case in practice. We assume that SUs also are needed to satisfy heterogenous constraints with different positions by taking the fairness of SUs into account. Moreover, we develop a weighted robust distributed power allocation scheme to guarantees the fairness of SUs. Techniques from robust optimization theory are used to study an objective formulation with infinite numbers of constraints. The optimal solution is obtained by using Lagrange dual method, and the Lagrange multiplier is solved by the sub-gradient update algorithm. The feasibility and availability of our proposed power allocation scheme is confirmed by numerical results.
\end{abstract}

Keywords - Cognitive radio networks, power control, fairness of SUs, robust optimization

\section{Introduction}

With the development of cognitive technology, the last decade has witnessed a growing demand for wireless radio spectrum. The concept of cognitive radio is proposed to address the issue of spectrum efficiency and has been receiving an increasing attention in recent years, since it equips SUs the capability to optimally adapt their operating parameters according to the interactions with the surrounding radio environment [1].The Federal Communications Commission (FCC) found the utilization of the spectrum resource is low most of time. Thus, the technology of cognitive radio networks is proposed to solve the problem of spectrum scarcity and improve spectrum efficiency.

In the cognitive radio networks, there are two approaches for dynamic spectrum sharing, spectrum underlay and spectrum overlay. The overlay approach increases the spectrum efficiency by granting unused frequency bands of PUs when the frequency 
bands are sensed being unused in temporal and spatial domains, i.e., SUs can utilize TV White Space (TVWS) spectrum resource by adapting transmission parameters dynamically at a particular time in a particular geographic area [2]-[5]. However, in overlay schemes, the SUs transmit power with no severe restrictions on transmission power due to any interference from SU transmissions will not be harmful to the inactive PUs. The overlay approach may not utilize the characteristics of co-channel interference in cellular environment and wideband signaling such as wideband codedivision multiple access and ultra-wideband that can coexist with other systems because of low inter-user interference [6]. In the underlay approach, SUs share the licensed spectrum with PUs, so as not to cause any harmful interference to PUs [7][9].The approach prevents us from increasing the data rate of the SUs when the PUs become active, this could lead to further improvement in spectrum efficiency.

Prevalent works on power allocation in underlay mode are investigated to reduce the energy consumption and to maximize the energy-efficient and the cognitive network capacity [10]-[13]. In [10], an optimal set for multi-band multi-antenna cognitive radio networks is designed to minimize the sum power of SUs. In [11], a distributed algorithm is introduced that aims at minimization of the total transmit power by PUs and SUs link. However, the PUs are allowed to increase their transmission power without bounds. In [12], the authors study the hierarchy in energy games for cognitive radio networks. The problem is to maximize the energy-efficiency for each selfish SU. In [13], the author focus on the throughput maximization of spectrum sharing cognitive radio networks, and a novel receiver and frame structure that distinctly improves their achievable throughput. In all these works, the main concerns can be summarized to find the optimal solution under the consideration of many practical limitations, which do not consider the fairness of SUs in the practical system.

We realize that, besides satisfying many practical limitations to guarantee reliable communication in cognitive radio networks, different SUs also require to satisfy heterogenous constraints with different positions. If SUs transmitter is located close to the PUs receiver, SUs can possibly transmit power while keeping the interference to the PU within a small limit as explained in the next subsection through (1). That is to say, the weighted coefficient is small in this case. Besides, when each SU utilizes underutilized spectrum resource with regard to the same quality of service (QoS) in underlay paradigm, the weighted coefficient under proposed scheme increases with the augment of the distance between SUs transmitter and PUs receiver. In [14], a comprehensive analysis has been presented including how to quantify the interference power limits and how to affect the PUs by the used interference power model.

In this paper, we investigate a robust transmit power allocation problem for the underlay cognitive radio networks. Our target is to minimize the overall transmit power of the cognitive system with respect to the budget power of SUs and the interference power to the PUs as well as the QoS of the SUs, considering the fairness among the SUs. The weights parameters are assigned according to the distance from SUs to PUs. The closer the distance, the smaller the weighted coefficient of distribution in order to guarantee an acceptable level of transmit power in such environment. In contrast, SUs increase their transmit power through a high interference power threshold, in which a larger weighted coefficient is assigned to SU. In this case, not only the fairness of 
communication between SUs is ensured, but also cognitive system can achieve more green communication.

The rest of the paper is organized as follows. In section 2, we introduce the system model and the minimum total transmit power allocation (MTTPA) problem, and transfer the MTTPA problem into a robust formulation problem. In section 3, a weighted robust distributed power allocation algorithm is proposed, and Lagrange dual method [15] is introduced to solve above problem. In section 4, the performance of our proposed scheme is evaluated. Finally, we draw the conclusions in section 5.

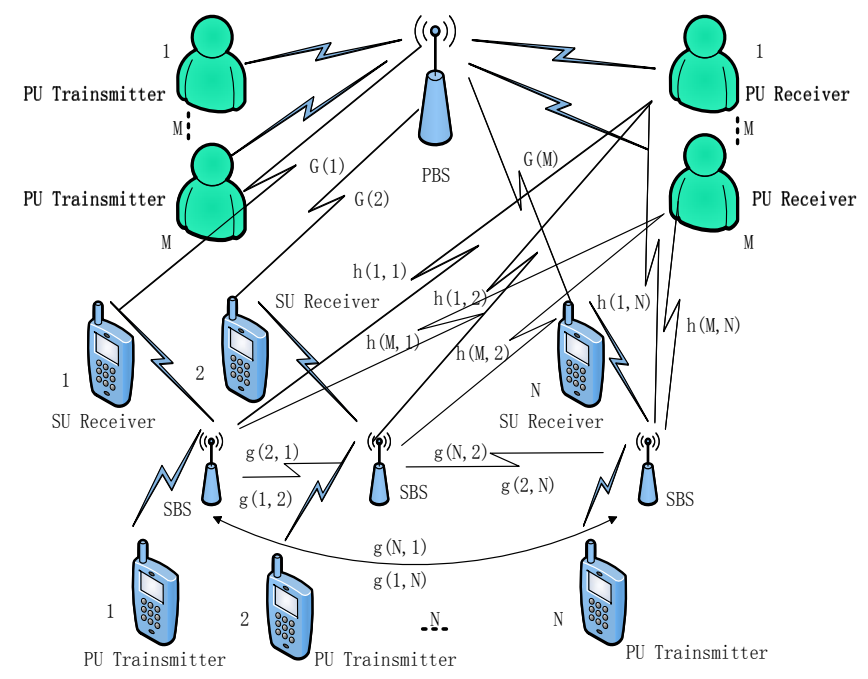

Fig. 1. System Model

\section{System Model and Problem Formulation}

In this section, we firstly describe the system model. Then, we provide the MTTPA problem scheme for the robust formulation in the cognitive radio networks.

\subsection{System Model}

This paper considers an ad-hoc cognitive radio network (AHCRN) as shown in Fig.1, which consists of multiple distributed cognitive links. PBS and SBS refer to the PUs' base station and SUs' base station, respectively. Both $G, g$ and $h$ represent channel gain between users. Each link consists of a transmitter node and a receiver node. Consider the AHCRN with $M$ SUs, coexisting with $N$ PUs in a licensed system. In order to further expand the scope of application of the distributed resource allocation method, we consider the fairness of resource allocation among SUs. It is assumed that each SU can acquire its location information perfectly by sensing method. And these information are sent to the corresponding transmitter via a feedback channel. 
As mentioned in the introduction, we consider the underlay paradigm where the instantaneous interference power introduced to the PUs receiver should below a certain threshold. Considering the fairness of the SUs, we have

$$
h_{i} p_{i} \leq \wp_{i} I_{t h}, \forall i \in\{1,2, \ldots, M\}
$$

where $\sum_{i} \wp_{i}=1, \wp_{i}$ is a weighted coefficient, $I_{t h}$ is the tolerable interference power threshold, $h_{i}$ is the channel gain from SU transmitter $i$ to PU receiver and $p_{i}$ represents the transmit power of SU transmitter $i$.

In order to administrate the interference among SUs and avoid intolerable interference to PUs, SUs cannot increase its total transmission power beyond a certain level. Considering the fairness of the SUs, we have

$$
p_{i} \leq \wp_{i} p_{T}, \forall i \in\{1,2, \ldots, M\}
$$

that is, the amount of SUs' power should not exceed $p_{T}$, where $p_{T}$ is the total power budget of SUs.

To guarantee SUs' QoS, the SINR of each SU must be no less than a certain target value to guarantee a reliable transmission. Thus, the SINR should be restricted by

$$
R_{i}{ }^{S I N R} \leq R_{i}^{\text {tar }}, \forall i \in\{1,2, \ldots, M\}
$$

where $R_{i}{ }^{S I N R}$ is the SINR of SUi, $R_{i}^{\text {tar }}$ is theminimum SINR requirement. And the $R_{i}{ }^{S I N R}$ can be represented by

$$
R_{i}{ }^{S I N R}=\frac{P_{i} g_{i i}}{\sum_{j \neq i} P_{j} g_{i j}+I_{p i}+\sigma_{i}^{2}}, \forall i \in\{1,2, \ldots, M\}
$$

where $g_{i i}$ represents the channel gain from SU transmitterito SU receiver $i$ and $g_{i j}$ represents the channel gain from SU transmitter $j$ to SU receiver $i, p_{j}$ represents the transmit power of SU transmitter $j$. And, $I_{p i}$ is interference power caused by PU to SU $i$, $I_{p i}=p_{0} G_{i}, p_{0}$ represents the transmit power of PU transmitter, $G_{i}$ represents the channel gain from PU transmitter to SU receiver $i$, and $\sigma_{i}$ is the background noise.

The SINR requirement problem can be written as follows

$$
\frac{N_{i}}{P_{i} g_{i i}} \leq \frac{1}{R_{i}^{t a r}}
$$

where $N_{i}=\sum_{j \neq i} P_{j} g_{i j}+I_{p i}+\sigma_{i}^{2}$.

\section{$2.2 \quad$ MTTPA Problem}

The objective is to design a MTTPA problem under the constraints of the interference power threshold from PU, the transmitting power budget of each SU and the SINR requirement of each SU. The optimization problem is formulated as

$$
\begin{gathered}
\min \sum_{i}^{M} p_{i} \\
\text { s.t. } C 1: p_{i} \leq \wp_{i} p_{T}, \forall i \in\{1,2, \ldots, M\}
\end{gathered}
$$




$$
\begin{aligned}
& C 2: h_{i} p_{i} \leq \wp_{i} I_{t h}, \forall i \in\{1,2, \ldots, M\} \\
& C 3: \frac{N_{i}}{P_{i} g_{i i}} \leq \frac{1}{R_{i}^{\text {tar }}}, \forall i \in\{1,2, \ldots, M\}
\end{aligned}
$$

\subsection{Robust Formulation}

In this section, we consider the uncertainty in channel gain matrix due to fluctuation of the channels. The specific shape of the uncertainty set depends on the underlying channel model and the sources of uncertainty. Then, we use ellipsoid to characterize the uncertainty set of channel gain, which succinctly describe a set of discrete points in Euclidean geometry.

The interference channel gain denotes $\mathfrak{I}=\left[\mathfrak{J}_{i}\right]$, which describes the perturbation of interference channel gain at link $i$. In order to incorporate the effect of imperfect channel gain, we write $h_{i}=\bar{h}_{i}+\Delta h_{i}$, where $\bar{h}_{i}$ is the nominal value. And $\Delta h_{i}$ means the channel error, which is the perturbation part. Using the additive uncertainty description method, we can obtain an ellipsoidal set to describe the uncertainty of interference channel gains as

$$
\mathfrak{I}_{i}=\left\{h_{i} \mid \bar{h}_{i}+\Delta h_{i}:\left\|\Delta h_{i}\right\|_{2}^{2} \leq \varepsilon_{h}^{2}, \forall i \epsilon\{1,2, \ldots, M\}\right.
$$

where $\|\cdot\|$ refers to the Euclidean norm, and $h_{i}$ is theithrow of $\Im$ and $\Delta h_{i}$ is the perturbation part. $\varepsilon_{h} \geq 0$ denotes the upper bound on the ellipsoidal set.

$\alpha_{i j}$ is determined by

$$
\alpha_{i j}=\frac{g_{i j}}{g_{i i}}, \forall j \neq i, \forall i \in\{1,2, \ldots, M\}
$$

Similarly, the channel gain $\alpha_{i j}$ is written as $\alpha_{i j}=\bar{\alpha}_{i j}+\Delta \alpha_{i j}$, with $\bar{\alpha}_{i j}$ represents the nominal value of $\alpha_{i j}$, and $\Delta \alpha_{i j}$ is the corresponding perturbation part. Besides, $\alpha_{i j}$ can capture the perturbation of interfering channel gains relative to the main channel gain of link $i$. Then the uncertainty set under ellipsoid approximation can be represented by

$$
\aleph_{i}=\left\{\alpha_{i j} \mid \bar{\alpha}_{i j}+\Delta \alpha_{i j}:\left\|\Delta \alpha_{i j}\right\|_{2}^{2} \leq \varepsilon_{\alpha}^{2}, \forall j \neq i, \forall i \in\{1,2, \ldots, M\}\right.
$$

where $\aleph_{i}$ describes the perturbation of channel gains, and $\varepsilon_{\alpha} \geq 0$ is the upper bound on the ellipsoidal set.

$\varepsilon_{h}$ and $\varepsilon_{\alpha}$ denote the size of uncertainty region respectively. The bigger $\varepsilon_{h}$ and $\varepsilon_{\alpha}$ is, the more uncertainty there is in channel. Based on the ellipsoidal sets (7) and (9), the weighted robust distributed power allocation problem can be formulated as following

$$
\begin{array}{cl} 
& \min \sum_{i}^{M} p_{i} \\
\text { s.t. } & C 1: p_{i} \leq \wp_{i} p_{T}, \forall i \in\{1,2, \ldots, M\} \\
& C 2:\left(\bar{h}_{i}+\Delta h_{i}\right) p_{i} \leq \wp_{i} I_{t h}, \forall i \in\{1,2, \ldots, M\}
\end{array}
$$




$$
C 3: \frac{\left\{\sum_{j \neq i}\left(\bar{\alpha}_{i j}+\Delta \alpha_{i j}\right) p_{j}+\eta_{i} / g_{i i}\right\}}{P_{i}} \leq \frac{1}{R_{i}^{t a r}}, \forall i \in\{1,2, \ldots, M\}
$$

where $\eta_{i}=\left(\bar{G}_{i}+\Delta G_{i}\right) p_{0}+\sigma_{i}^{2}, \bar{G}_{i}$ represents the nominal value and $\Delta G_{i}$ is the corresponding perturbation part.

The formulation (10) is a minimization of concave function with linear constraints, which cannot be solved by standard convex optimization scheme due to the infinity of objective constraint C3. In other words, the formulation is subject to a NP-hard problem [16] respect to sets $\mathfrak{\Im}_{i}$, for all $i$ and $\aleph_{i}$.

\section{Weighted Robust Distributed Power Allocation Algorithm}

In this section, we present the weighted robust distributed power allocation algorithm, after introducing the worst estimation error and the Lagrange dual approach for problem formulation.

\subsection{Solution of the Optimization Problem}

The problem (10) belongs to a semi-infinite programming (SIP) owing to the constraint $\mathrm{C} 2$ and $\mathrm{C} 3$, which make it difficult to handle. In order to get the optimal transmit power, the problem (10) is transformed into a deterministic optimization problem under the condition of limited constraints. From Cauchy-Schwarz inequality, we have

$$
\sum_{j \neq i}\left|\bar{\alpha}_{i j} p_{j}\right|+\sum_{j \neq i}\left|\Delta \alpha_{i j} p_{j}\right| \leq \sum_{j \neq i}\left|\bar{\alpha}_{i j} p_{j}\right|+\left(\sum_{j \neq i}\left|\Delta \alpha_{i j}\right|^{2}\right)^{1 / 2}\left(\sum_{j \neq i}\left|\Delta p_{j}\right|^{2}\right)^{1 / 2}
$$

By applying (9), we introduce an equation in (11) based on the worst estimation error as follows

$$
\max _{\alpha_{i j} \in \mathrm{N}_{i}}\left\{\sum_{j \neq i}\left|\bar{\alpha}_{i j} p_{j}\right|+\sum_{j \neq i}\left|\Delta \alpha_{i j} p_{j}\right|\right\}=\sum_{j \neq i} \bar{\alpha}_{i j} p_{j}+\varepsilon_{\alpha} \sqrt{\sum_{j \neq i} p_{j}^{2}}
$$

Similarly, taking all parameters' uncertainties into consideration and from the perspective of worst case optimization, the weighted robust distributed power allocation problem is rewritten as follows

$$
\begin{gathered}
\min \sum_{i}^{M} p_{i} \\
\text { s.t. } C 1: p_{i} \leq \wp_{i} p_{T}, \forall i \epsilon\{1,2, \ldots, M\} \\
\text { C2: }\left(\bar{h}_{i}+\varepsilon_{h}\right) p_{i} \leq \wp_{i} I_{t h}, \forall i \in\{1,2, \ldots, M\} \\
\text { C3: } \frac{\left(\sum_{j \neq i} \bar{\alpha}_{i j} p_{j}+\varepsilon_{\alpha} \sqrt{\sum_{j \neq i} p_{j}^{2}}+\varphi_{i} / g_{i i}\right)}{P_{i}} \leq \frac{1}{R_{i}^{\text {tar }}}, \forall i \in\{1,2, \ldots, M\}
\end{gathered}
$$

where $\varphi_{i}=\left(\bar{G}_{i}+\mathcal{E}_{G}\right) p_{0}+\sigma_{i}^{2}, \mathcal{E}_{G}$ is the maximum uncertainty of the channel gain from $\mathrm{PU}$ transmitter to $\mathrm{SU}$ receiver. 


\subsection{Weighted Robust Distributed Algorithm}

We can see that the problem (13) is a convex optimization problem [16]. The optimal transmission power for this problem can be calculated by Lagrange dual approach. To apply the dual decomposition to the problem (13), additional auxiliary variables and corresponding equality constraints need to be introduced owing to the specific nature of problem, in which the coupling exists in both objective functions and the system wide constraints [17]. According to the Lagrange principle, the Lagrange function as follows

$$
\begin{gathered}
L\left(\left\{p_{i}\right\},\left\{\lambda_{i}\right\},\left\{u_{i}\right\},\left\{v_{i}\right\}\right)=\sum_{i} p_{i}+\sum_{i} \lambda_{i}\left(p_{i}-\wp_{i} p_{T}\right) \\
+\sum_{i} u_{i}\left(\left(\bar{h}_{i}+\varepsilon_{h}\right) p_{i}-\wp_{i} I_{t h}\right)+\sum_{i} v_{i}\left(\frac{\left(\sum_{j \neq i} \bar{\alpha}_{i j} p_{j}+\varepsilon_{\alpha} \sqrt{\sum_{j \neq i} p_{j}^{2}}+\varphi_{i} / g_{i i}\right)}{P_{i}}-\frac{1}{R_{i}^{t a r}}\right) \\
\forall i \in\{1,2, \ldots, M\}
\end{gathered}
$$

where $\lambda_{i} \geq 0, u_{i} \geq 0$ and $v_{i} \geq 0$ are the Lagrange multipliers.

Due to the powers of SUs are coupled above problem, the problem can be decomposed into the minimization power problem and the dual optimization problem of Lagrange multipliers by the Lagrange dual decomposition method. The dual function of (14) can be expressed as:

$$
\begin{aligned}
\Psi\left(\left\{\lambda_{i}\right\},\left\{u_{i}\right\},\left\{v_{i}\right\}\right)=\min _{p_{i}} \sum_{i}^{M} L_{i}\left(\left\{p_{i}\right\},\left\{\lambda_{i}\right\},\left\{u_{i}\right\},\left\{v_{i}\right\}\right) \\
-\sum_{i}^{M}\left\{\lambda_{i} \wp_{i} p_{T}+u_{i} \wp_{i} I_{\text {th }}+\frac{v_{i}}{R_{i}^{\text {tar }}}\right\}
\end{aligned}
$$

where each sub-optimization problem $L_{i}$ represents the resource allocation problem in each SU link since the decomposability of the Lagrange function. We have

$$
\begin{aligned}
L_{i}\left(\left\{p_{i}\right\},\left\{\lambda_{i}\right\},\left\{u_{i}\right\},\left\{v_{i}\right\}\right) & =p_{i}+\lambda_{i} p_{i}+u_{i}\left(\bar{h}_{i}+\varepsilon_{h}\right) p_{i} \\
& +v_{i} \frac{\left(\sum_{j \neq i} \bar{\alpha}_{i j} p_{j}+\varepsilon_{\alpha} \sqrt{\sum_{j \neq i} p_{j}^{2}}+\varphi_{i} / g_{i i}\right)}{P_{i}}, \forall i \in\{1,2, \ldots, M\}
\end{aligned}
$$

By applying dual decomposition method, we obtain the corresponding dual problem of (15) is

$$
\begin{gathered}
\max \psi\left(\left\{\lambda_{i}\right\},\left\{u_{i}\right\},\left\{v_{i}\right\}\right) \\
\text { s.t. } \lambda_{i} \geq 0, u_{i} \geq 0, v_{i} \geq 0, \forall i \in\{1,2, \ldots, M\}
\end{gathered}
$$

According to the sub-gradient updating algorithm, the Lagrange multipliers updating function as follows

$$
\begin{aligned}
& \lambda_{i}(t+1)=\left[\lambda_{i}(t)+S_{-} \lambda_{i} * \alpha\right]^{+}, \forall i \in\{1,2, \ldots, M\} \\
& u_{i}(t+1)=\left[u_{i}(t)+S_{-} u_{i} * \beta\right]^{+}, \forall i \in\{1,2, \ldots, M\}
\end{aligned}
$$




$$
v_{i}(t+1)=\left[v_{i}(t)+S_{-} v_{i} * \gamma\right]^{+}, \forall i \in\{1,2, \ldots, M\}
$$

where $\alpha, \beta$, and $\gamma$ arestep-size, $[X]^{+}=\max \{X, 0\}$ and $t$ is time gap. The corresponding Lagrange sub-gradient $S_{-} \lambda_{i}, S_{-} u_{i}$, and $S_{-} v_{i}$ can then be expressed as:

$$
\begin{aligned}
& S_{-} \lambda_{i}=p_{i}-\wp_{i} p_{T}, \forall i \epsilon\{1,2, \ldots, M\} \\
& S_{-} u_{i}=\left(\bar{h}_{i}+\varepsilon_{h}\right) p_{i}-\wp_{i} I_{t h}, \forall i \epsilon\{1,2, \ldots, M\} \\
& S_{-} v_{i}=\frac{\left(\sum_{j \neq i} \bar{\alpha}_{i j} p_{j}+\varepsilon_{\alpha} \sqrt{\sum_{j \neq i} p_{j}^{2}}+\varphi_{i} / g_{i i}\right)}{P_{i}}-\frac{1}{R_{i}^{\operatorname{tar}}}, \forall i \epsilon\{1,2, \ldots, M\}
\end{aligned}
$$

Based on the above description and deduction, the optimal transmit power of each SU transmitter can be obtained individually according to the Karush Kuhn Tucker (KKT) conditions [18] through the following equation:

$$
\frac{\partial L_{i}\left(\left\{p_{i}\right\},\left\{\lambda_{i}\right\},\left\{u_{i}\right\},\left\{v_{i}\right\}\right)}{\partial P_{i}}=0
$$

and the optimal solution of robust power control scheme for a given $\lambda_{i}, u_{i}, v_{i}$ is

$$
P_{i}^{o p t}=\sqrt{\frac{v_{i}\left(\sum_{j \neq i} \bar{\alpha}_{i j} p_{j}+\varepsilon_{\alpha} \sqrt{\sum_{j \neq i} p_{j}^{2}}+\varphi_{i} / g_{i i}\right)}{1+\lambda_{i}+u_{i}\left(\bar{h}_{i}+\varepsilon_{h}\right)}}, \forall i \in\{1,2, \ldots, M\}
$$

We adopt sub-gradient method to solve the dual problem (17). Since the subgradient method is able to converge to the dual optimal solution, we can obtain the optimal the power control solution through (21). To apply the optimal solution, it needs a central controller that obtains the location distribution information and the number of SUs by sensing method, and broadcasts Lagrange multipliers $\lambda_{i}(t+$ $1), u_{i}(t+1), v_{i}(t+1)$ and $p_{i}{ }^{\text {opt }}(t+1)$. Such a system may not be practical because of infrastructure coat, or the difficultly to build control channel to exchange parameters information among SUs. Therefore, we derive a distributed mechanism in AHCRN that can utilize the inherent PU feedback information. We summarize the weighted robust distributed power allocation algorithm as below.

Algorithm: Weighted Robust Distributed Power Allocation

1. Initialization variables:

$0 \leq p_{i}{ }^{o p t}(0) \leq \wp_{i} p_{T}, \lambda_{i}(0) \geq 0, u_{i}(0) \geq 0, v_{i}(0) \geq 0, \alpha \geq 0, \beta \geq 0, \gamma \geq 0$

2. Calculation variables:

i)Calculate the interference generated by SUs according to constraint C2 in (13), and calculate the $\operatorname{SINR} R_{i}{ }^{S I N R}$ by (4); Calculate the interference $\varphi_{i}$ by PUs according to (13), where $\sigma_{i}$ is generated by random function;

ii) Calculate $\lambda_{i}(t+1) \geq 0, u_{i}(t+1) \geq 0, v_{i}(t+1) \geq 0$ and $S_{-} \lambda_{i}, S_{-} u_{i}, S_{-} v_{i}$ according to (18) and (19), respectively;

3. Update the SU strategy simultaneously:

$\lambda_{i}(t+1), u_{i}(t+1), v_{i}(t+1)$;

$p_{i}{ }^{o p t}(t+1)=\min \left\{p_{i}{ }^{o p t}(t), \wp_{i} p_{T}\right\}$, where $p_{i}{ }^{o p t}(t)$ is calculated by (21);

4. Go to step 2 . 


\section{Simulation Results and Discussion}

\subsection{Simulation Parameters and Assumptions}

The numerical results of the proposed weighted robust distributed power allocation algorithm are presented in this section. Our numerical experiments are performed to support the theoretical discussions of previous sections. In order to illustrate the effectiveness and convergence of the algorithm, and the convenience of calculation mathematically, we consider the scenario in the AHCRN, wherein three SUs and one PU share the same spectrum with each other. The value of $p_{T}, p_{0}$ and $I_{t h}$ are assumed to be $1.7 \mathrm{~mW}, 2 \mathrm{~mW}$ and $0.3 \mathrm{~mW}$, respectively. The background noise $\sigma_{i}$ is generated by random function, and the interval of random function is $(0,0.1)$. The minimum SINR requirement values $R_{i}^{\text {tar }}$ are assumed to be $6.85 \mathrm{~dB}, 7.60 \mathrm{~dB}$ and $8.29 \mathrm{~dB}$, respectively. Furthermore, the weighted constraint values $\wp_{i}$ are assumed to be $0.25,0.35$ and 0.40 .

\subsection{Performance Analysis}

In Fig.2, simulation results are provided to evaluate the interference performance of the proposed weighted robust scheme (PWRS) compared with the proposed weighted non-robust scheme (PWNRS) under the AHCRN. The PWNRS refers to the scheme which does not take channel uncertainties into account and directly utilizes the estimates of all channels. Similarly, the PWRS investigates the scheme in practical system that the perturbation value $\varepsilon$ is assumed to be $5 \%, 7.5 \%, 10 \%$, respectively. And $\varepsilon$ include three parts, $\varepsilon_{h}, \varepsilon_{\alpha}$ and $\varepsilon_{G}$.

In this scenario, the interference incurred by SUs has the lowest level which can guarantee constraint $\mathrm{C} 2$ in (13) under PWNRS, but it may be violate this constraint when they adopt the optimal transmit power to achieve MTTPA without considering the uncertainty in practical system. In order to show the effect of parameter's uncertainty on interference more clearly, we change the uncertainties of parameters. In Fig.2, the PWRS ( $\varepsilon=5 \%, \varepsilon=7.5 \%)$ can guarantee constraint $\mathrm{C} 2$ in (13) while the PWRS $(\varepsilon=10 \%)$ violates this constraint.

In Fig.3, we can also obtain that the interference to PU increases linearly with parameter perturbation for PWRS because the algorithm requires more transmit power to suppress the impact of parameter perturbation on the system, which guarantee that the actual SINR of SU receiver is not below the target value. This phenomenon shows the trade-off between performance (i.e., the interference to PU) and robustness $(\varepsilon)$.

In Fig.4 (a), simulation results are provided to evaluate the SU3' SINR of PWRS compared with PWNRS under the AHCRN, $R_{3}{ }^{\text {tar }}$ is the SINR of SU3. As we see, the PWNRS and PWRS can guarantee the SINR of SU3 no less than the minimum target SINR, but the PWRS below the PWNRS owing to the parameter perturbation. In Fig.4 (b), SUs' SINR under PWRS with the variation of $\varepsilon$ is evaluated. SUs' SINR decreases with the growing of $\varepsilon$, since the SINR is no-increasing function with respect to the parameters' perturbations. The larger $\varepsilon$ means that there is a lager $\varepsilon$ perturbation in the system. This phenomenon shows the trade-off between SUs' SINR and 
robustness $(\varepsilon)$. Thus, we can confirm that the PWRS is efficient compared with the PWNRS.

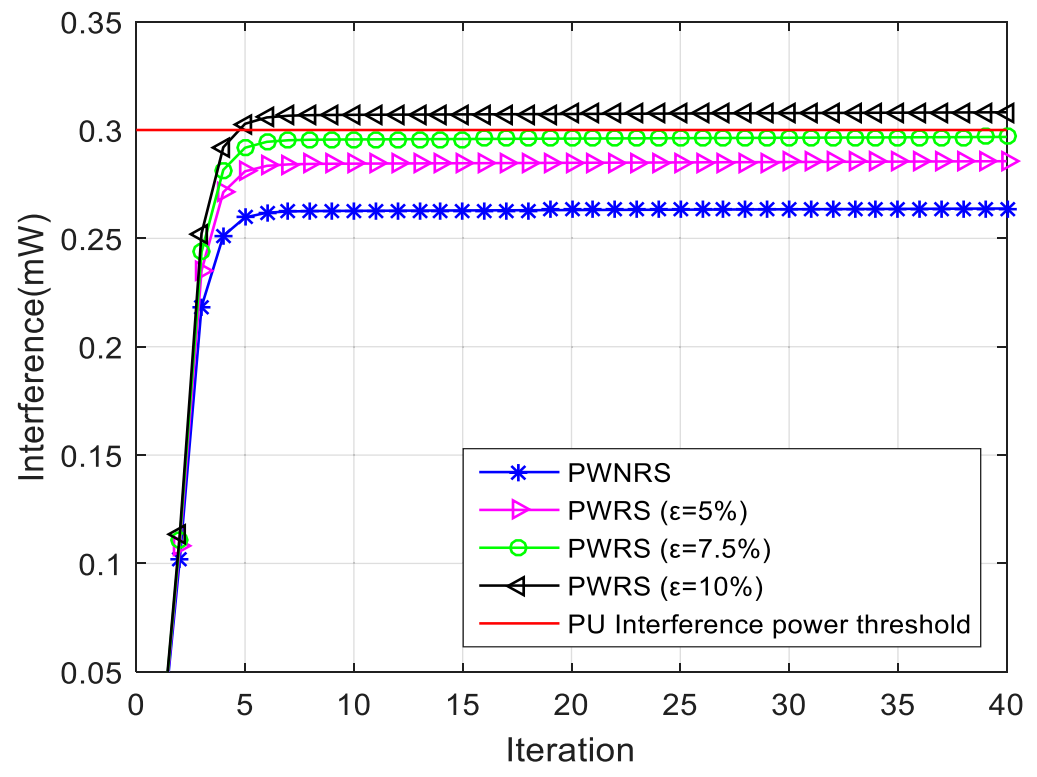

Fig. 2. The interference to the PU under PWNRS and PWRS

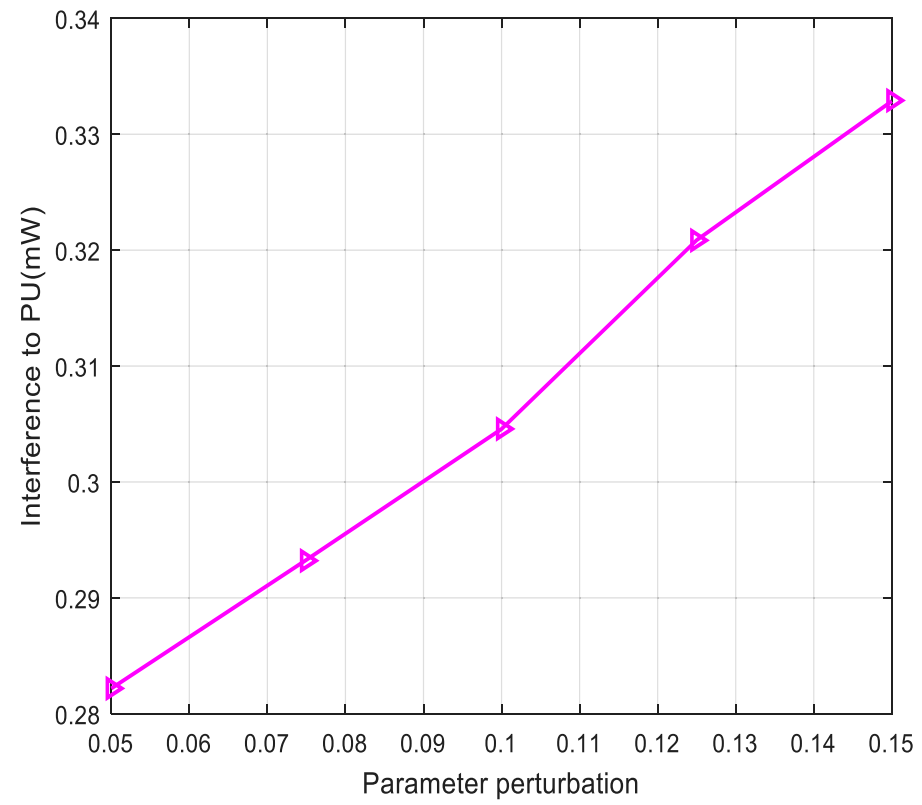

Fig. 3. Parameter perturbation $\varepsilon$ versus Interference to PU under PWRS 


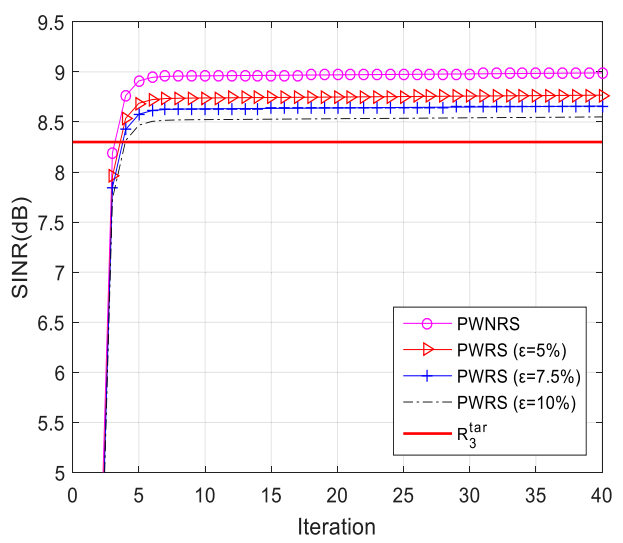

(a)The SINR of SU for different schemes

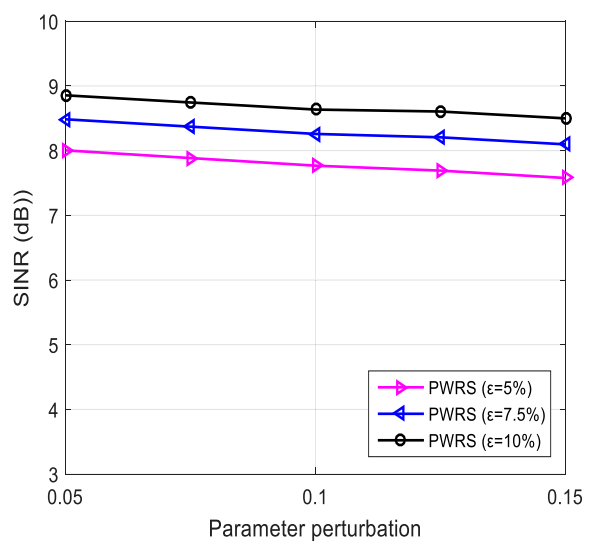

(b) Parameter perturbation $\varepsilon$ versus SINR for PWRS

Fig. 4. The SINR of SU3 under different schemes and Parameter perturbation $\varepsilon$ versus SINR under PWRS

In Fig.5, we compare the total power, the capacity, the interference performance and the SINR experienced by SUs of different schemes: PWRS and traditional robust scheme (TRS) [19]-[21] under the same parameter perturbation $(\varepsilon=5 \%)$.

Fig.5 (a) and Fig.5 (b) show histograms with the total power and total capacity of SUs with different schemes. We note that, with the increasing of total power for above schemes, the total power for $\operatorname{PWRS}(\varepsilon=5 \%)$ is greatly reduced when compared with TRS as in (1) and (2) which do introduce weights into its constraints. And the total power of TRS has reached the total power limit $\left(p_{T}=1.7 \mathrm{~mW}\right)$. It is found that the total power of PWNRS is the smallest, which means that it consumes minimal energy under the same data transmission, and the total of PWRS increase with the augment of $\varepsilon$. For the dynamic cognitive radio communication system, the PWNRS is obviously powerless. These two examples point that the PWRS consumes smallest power and the consumed power is increased with the disturbance amplitude. Similarly, we found that the capacity of PWNRS and TRS are larger than the capacity of PWRS as in Fig.5 (b).

Fig.5 (c) and Fig.5 (d) show the interference performance and the SINR of SUs for both PWRS and TRS. We note that the SINR of PWRS no more than the SINR of TRS as in Fig.5(d), which can not only ensure the seamless communication of the system, but also improve the energy efficiency of the system. The interference caused by SUs for TRS is greatly increased when compare with PWRS as in Fig.5(c). Furthermore, the interference of TRS achieves nearly $80 \%$ more than the interference power threshold.

A good candidate energy efficiency function is one that measure the system throughout for unit-energy consumption [22]. In [23]-[25], the energy efficiency function is the cost of users to gain their benefits or the QoS of the network. Fig.6 also shows the value of energy efficiency function (the sum of reliable data transmission divided by the total of transmit power consumed) toward increasing the parameter 


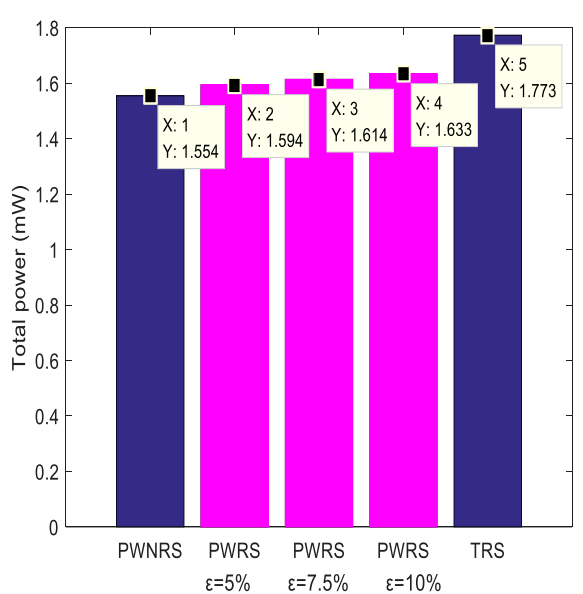

(a) The performance comparison of total power (b) The performance comparison of total capacity

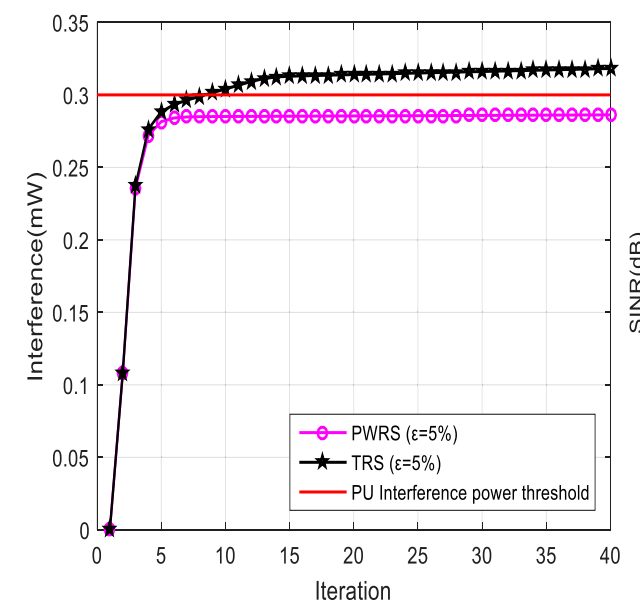

(c)The performance comparison of interference
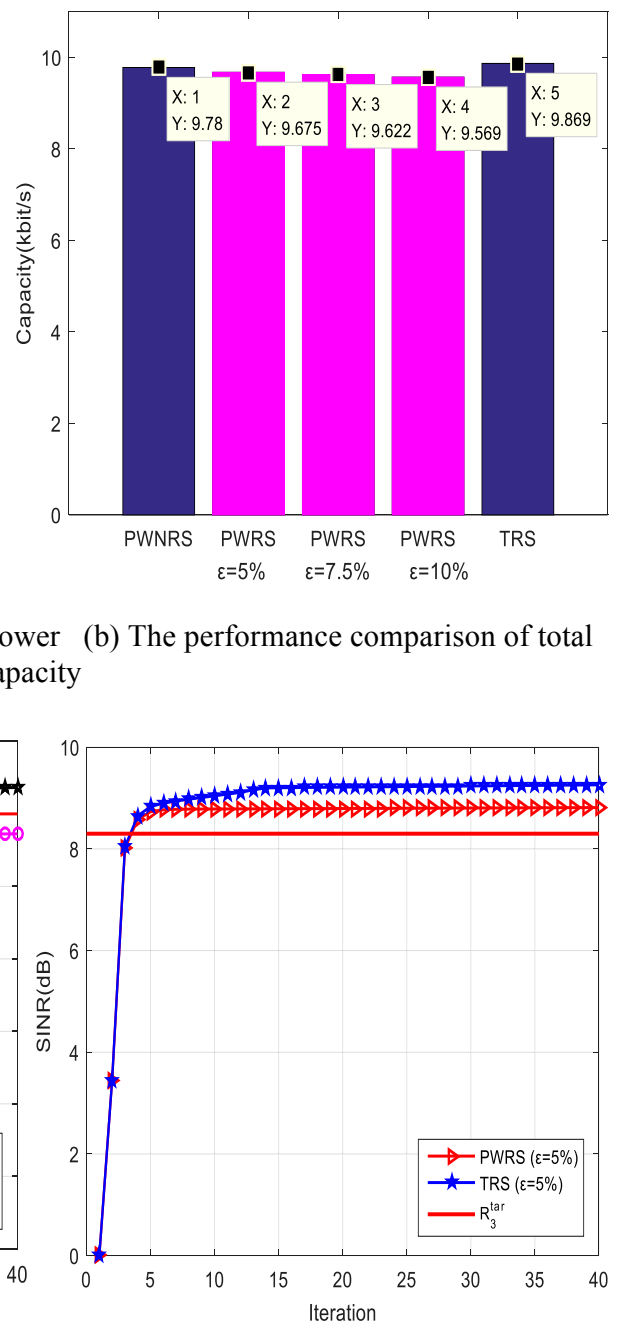

(d) The performance comparison of SINR

Fig. 5. The performance comparison of the algorithms, including the total power, the capacity, the interference and the SINR

perturbation. It can be observed that energy efficiency function for cognitive system is decreased by increasing the parameter perturbation. Therefore their SINR also decrease, which means the throughput will be decreased considerably as in Fig.5 (b). We can obtain that the energy efficiency function of PWRS is always located above the TRS because the TRS ignores the fairness of communication among SUs, indicting our proposed algorithm performs quit well for the consider problem. While ensuring the fairness of the communication, the strategy also can prevent some edge users increasing the transmission power. Hence reducing the energy consumption, we can achieve a more green communication. 


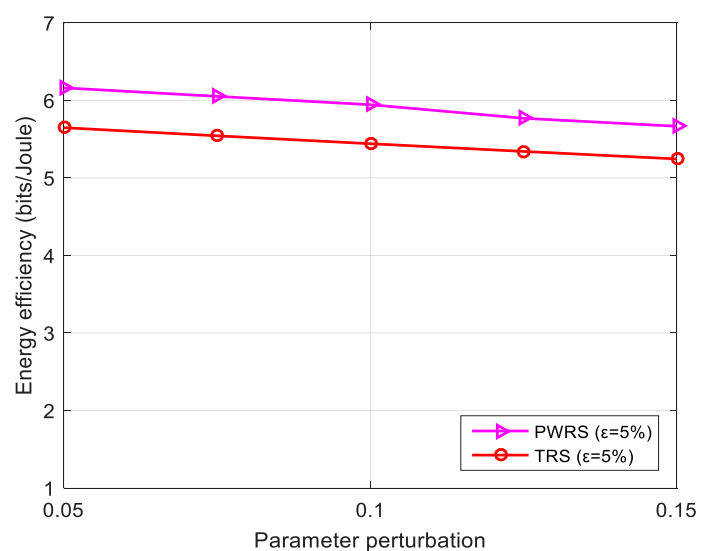

Fig. 6. The parameter perturbation $\varepsilon$ versus energy efficiency under different schemes

\section{Conclusions}

In this paper, we propose a weighted robust distributed power allocation algorithm based on underlay paradigm in ad-hoc cognitive radio networks. Our model is general and covers many practical constrains, including total transmission power constraint, the interference power constraint and required SINR constraint. Simulation results show that PWRS can achieve more rational allocation of power for resource sharing compared with other algorithms since we take the information state and the fairness of SUs into account. This scheme allows SUs consume less power to attain the basic SINR requirements. Either total transmission power or interference caused by SUs is decreased when compare with other algorithm without affecting the performance of the primary network. Additionally, the significant promotion in the energy efficiency of PWRS gives the highest preference to apply it in cognitive radio senor networks and green cognitive radio networks.

\section{Acknowledgment}

This work was supported by the Nation Natural Science Foundation of China (Grant NO.61501059) and the Education Department of Jilin Province (Grant NO.2016343). We would like to thank the anonymous reviewers and the editor, whose invaluable comments helped improve the presentation of this paper substantially.

\section{$7 \quad$ References}

[1] Haykin, S. (2005). Cognitive radio: brain-empowered wireless communications. IEEE Journal on Selected Areas in Communications, 23: 201-220. https://doi.org/10.1109/JSAC. $\underline{2004.839380}$ 
Paper-A Robust Power Allocation Scheme in Ad-Hoc Cognitive Radio Networks

[2] Cacciapuoti, A. S., Caleffi, M., Marino, F., \&Paura, L. (2016). Mobile smart grids: exploiting the tv white space in urban scenarios. IEEE Access, 4:7199-7211. https://doi.org/10.1109/ACCESS.2016.2620564

[3] Ding, G., Wang, J., Wu, Q., Yao, Y. D., Song, F., \&Tsiftsis, T. A. (2016). Cellular-basestation-assisted device-to-device communications in tv white space. IEEE Journal on Selected Areas in Communications, 34: 107-121. https://doi.org/10.1109/JSAC.2015. 2452532

[4] Caleffi, M., Cacciapuoti, A. S. (2016). Optimal database access for tv white space. IEEE Transactions on Communications, 64:83-93. https://doi.org/10.1109/TCOMM.2015.2498 607

[5] Cacciapuoti, A. S., Caleffi, M. (2015). Interference analysis for secondary coexistence in tv white space. IEEE Communications Letters, 19: 383-386. https://doi.org/10.1109/LCO MM.2014.2386349

[6] Dong, I. K., Long, B. L., \& Hossain, E. (2008). Joint rate and power allocation for cognitive radios in dynamic spectrum access environment. IEEE Transactions on Wireless Communications, 7: 5517-5527. https://doi.org/10.1109/T-WC.2008.071465

[7] Stotas, S.,Nallanathan, A. (2011). Enhancing the capacity of spectrum sharing cognitive radio networks. IEEE Transactions on Vehicular Technology, 60: 3768-3779. https://doi.org/10.1109/TVT.2011.2165306

[8] Sboui, L., Rezki, Z.,Alouini, M. S. (2015). Energy-efficient power allocation for underlay cognitive radio systems. IEEE Transactions on Cognitive Communications \& Networking, 1: 273-283. https://doi.org/10.1109/TCCN.2015.2488622

[9] Goldsmith, A., Jafar, S. A., Maric, I., Srinivasa, S. (2009). Breaking spectrum gridlock with cognitive radios: an information theoretic perspective. Proceedings of the IEEE, 97: 894-914. https://doi.org/10.1109/JPROC.2009.2015717

[10] Pan, C., Wang, J., Zhang, W., Du, B. (2014). Power minimization in multi-band multiantenna cognitive radio networks. Wireless Communications IEEE Transactions on, 13: 5056-5069. https://doi.org/10.1109/TWC.2014.2329296

[11] Islam, H., Liang, Y. C., Hoang, A. T. (2007). Distributed Power and Admission Control for Cognitive Radio Networks Using Antenna Arrays. IEEE International Symposium on New Frontiers in Dynamic Spectrum Access Networks (DySPAN), Dublin, Ireland. https://doi.org/10.1109/DYSPAN.2007.40

[12] Lasaulce, S., Hayel, Y., El Azouzi, R., Debbah, M. (2009). Introducing hierarchy in energy games. IEEE Transactions on Wireless Communications, 8: 3833-3843. https://doi.org/10.1109/TWC.2009.081443

[13] Wang, L., Sheng, M., Zhang, Y., \& Wang, X. (2015). Robust energy efficiency maximization in cognitive radio networks: the worst-case optimization approach. IEEE Transactions on Communications, 63: 51-65.

[14] Clancy, T. C. (2009). Dynamic spectrum access using the interference temperature model. Annals of Telecommunications, 64: 573-592. https://doi.org/10.1007/s12243-009-0098-x

[15] Tan, X., Zhang, H., Hu, J. (2013). Achievable transmission rate of the secondary user in cognitive radio networks with hybrid spectrum access strategy. IEEE Communications Letters, 17: 2088-2091. https://doi.org/10.1109/LCOMM.2013.091913.131637

[16] Le, L. B., Hossain, E. (2008). Resource allocation for spectrum underlay in cognitive radio networks. IEEE Transactions on Wireless Communications, 7: 5306-5315. https://doi.org/10.1109/T-WC.2008.070890

[17] Boyd, Vandenberghe, Faybusovich. (2004).Convex Optimization, Cambridge University Press.

[18] Palomar, D. P.,Chiang, M. (2006). A tutorial on decomposition methods for network utility maximization. IEEE Journal on Selected Areas in Communications, 24: 1439-1451. https://doi.org/10.1109/JSAC.2006.879350 
[19] Parsaeefard, S., Sharafat, A. R. (2013). Robust distributed power control in cognitive radio networks. IEEE Transactions on Mobile Computing, 12: 609-620. https://doi.org/10.1109/TMC.2012.28

[20] Gong, S., Wang, P., Liu, Y., Zhuang, W. (2013). Robust power control with distribution uncertainty in cognitive radio networks. IEEE Journal on Selected Areas in Communications, 31: 2397-2408. https://doi.org/10.1109/JSAC.2013.131125

[21] Huang, S., Liu, X., Ding, Z.Distributed power control for cognitive user access based on primary link control feedback, Conference on Information Communications (INFOCOM), San Diego, USA. https://doi.org/10.1109/INFCOM.2010.5461916

[22] Goodman, D., Mandayam, N. (2000). Power control for wireless data. IEEE Personal Communications, 7: 48-54. https://doi.org/10.1109/98.839331

[23] He, C., Li, G. Y., Zheng, F. C., You, X. (2014). Energy-efficient resource allocation in ofdm systems with distributed antennas. IEEE Transactions on Vehicular Technology, 63: 1223-1231. https://doi.org/10.1109/TVT.2013.2282373

[24] Ng, D. W. K., Lo, E. S., Schober, R. (2012). Energy-efficient resource allocation in multicell ofdma systems with limited backhaul capacity. IEEE Transactions on Wireless Communications, 11: 3618-3631. https://doi.org/10.1109/TWC.2012.083112.111951

[25] Alabbasi, A., Rezki, Z., Shihada, B. (2015). Energy efficient resource allocation for cognitive radios: a generalized sensing analysis. IEEE Transactions on Wireless Communications, 14: 2455-2469. https://doi.org/10.1109/TWC.2014.2387161

\section{Authors}

Hongzhi Wang was born in Heilongjiang Province, China, in 1961. He received the B.S. degree from Tianjin University, Tianjin, in 1983, in Electronic instruments and measurement techniques and M.S. degree from Jilin University of Technology, Jilin, in 1993, in communications and information systems. He received the Ph.D. degree from Jilin University, Jilin, in 2000, in communications and information systems. He is with the College of Computer Science and Technology, Changchun University of Technology, Changchun and 130012, China. His research interests include digital signal processing and application, image processing. (e-mail: hzwang@ccut.edu.cn).

Meng Zhu was born in Anhui Province, China, in 1991. He received the B.S. degree from West Anhui University, Luan, in 2015, in communication engineering. He is currently pursuing the M.S. degree with the Department of Computer Science and Engineering, the Changchun University of Technology, Changchun 130012, China. His research interests include cognitive radio, resource allocation, and signal processing. (e-mail: zhumengvipzm@163.com).

Mingyue Zhou (Corresponding author) was born in Jilin Province, China, in 1980. She received the B.S. and M.S. degrees both from Changchun University of Technology, Changchun, in 2004 and 2007 respectively, and the Ph.D. degree from Jilin University, Changchun 130012, China, in 2014. Her research interests include cognitive radio, resource allocation, and signal processing. (e-mail: zmyjlu@ccut.edu.cn).

Article submitted 17 March 2017. Published as resubmitted by the authors 27 June 2017. 